\title{
Impact of Peridontitis Education Program on Peridontitis Patients' Knowledge, Beliefs, and behavior in Riyadh City
}

\begin{abstract}
Ebtisam M. Fetohy*
Abstract: An intervention experimental study was conducted to assess the impact of Peridontitis education program (PEP) for Peridontitis patients in King Abdel Aziz University Hospital (KAAUH) in Riyadh City. The program was administered to 103 patients through one session and one immediate assessment. Another 103 patients formed the control group. The results showed that the mean knowledge score, the mean scores of the perceived seriousness ( $p$. seriousness), $p$. benefits, and $p$. barriers of intervention group were significantly higher than the control group. Stepwise multiple regression models revealed that total knowledge, perceived benefits, patients' work, and family size were predictors of patients' practice of control group $(\beta=0.287,0.218,0.194$, and 0.192$)$. Total knowledge, patients' work and total health beliefs model (HBM) score were predictors of patients' practice of the intervention group $\left(R^{2}=0.303\right)$. The study recommended the replication of such program and a more long term one to have more improvement in patients' knowledge, all beliefs, and practice.
\end{abstract}

Keywords; Peridontitis Education Program (PEP); Peridontitis Knowledge; Beliefs and Practices (KPP; Oral Hygiene)

\section{INTRODUCTION}

Oral diseases are clearly related to behavior, and the prevalence of dental caries and periodontal disease has decreased with improvement in the oral hygiene and the decrease in the consumption of sugar product. This general favorable trend in reducing oral diseases, however, has not been seen in several developing countries or in the Middle East.1,2 While twice-a-day tooth brushing seems to be an established practice in several industrialized countries such as United Kingdom,3 Italy,4 Sweden,5 and Norway, 6 this goal is still far from being realized in several other countries, including

\footnotetext{
*Health Administration \& Behavioral Sciences Dept., High Institute of Public Health, Alexandria, Egypt.
} 
Saudi Arabia,1 Turkey,7 Lebanon,8 and Kuwait. 9,10

In parallel to changing oral disease patterns, oral health awareness, dental knowledge, and positive dental health attitudes of the general public have grown.11-14 Oral health knowledge is considered to be an essential prerequisite for health related behavior, 15 although only a weak association seems to exist between knowledge and behavior in cross-sectional studies.16,17 Nevertheless, studies have shown that there is an association between increased knowledge and better oral health. ${ }^{18,19}$

The health belief model (HBM), is one of the first models to adapt theories from the behavioral sciences in order to examine health-related problems. It is still one of the most widely recognized and used models in health behavior applications.20 The four constructs of the model are perceived susceptibility, perceived seriousness, perceived benefits of taking action, and perceived barriers to taking action. ${ }^{20}$

There is a need for studies assessing changes in knowledge, beliefs, and practice of Saudi patients having peridontitis following the implementation of Peridontitis education program (PEP). Therefore, this study aimed at assessing the impact of short-term PEP for patients having the disease. The PEP aims at increasing patients' knowledge, improving beliefs to compliance with treatment and healthy practices and increasing compliance, and inducing positive behavior changes.

\section{MATERIAL and METHODS:}

Study design, settings, and population:

The study population was the outpatients having peridontitis at King Abdel Aziz University Hospital (KAAUH) and agreed to participate in the study. Two hundred and six patients: 103 patients were subjected for PHE intervention and the other 103 were controls. The control group patients were interviewed first to prevent information pollution. At each working day, 
a group of patients of the intervention group having peridontitis who agreed to participate were collected in a staff room to attend the session. Each group number ranged from 8-12. Ten groups were subjected to PEP. Each session of the program took about 2 hours for each group. The patients of the intervention group were interviewed by the questionnaire immediately after the session.

\section{Peridontitis Education Program (PEP):}

The objectives of PEP were encouraging the improvement in compliance with treatment recommendations. This improvement in compliance could be happened by increasing knowledge and improving beliefs.

\section{Methods of teaching:}

Lectures, group discussion, and role-play were used. Visual aids in the form of posters, handout, and pamphlets were used. Two health educators in the hospital helped the researcher in the presentation of contents of the program and the interview. The contents were presented in a simple form to suit all educational levels of the patients, with the emphasis on benefits of compliance and good oral health practice rather than harmful effects of non-compliance

\section{Contents of the program}

1- General information about definition of peridontitis, signs and symptoms, causes, risk factors, and complications.

2- Benefits of early detection and compliance with treatment and how to overcome barriers to compliance.

3-Treatment options available, how to do tooth and tongue brushing, flossing, and mouth wash

\section{Evaluation procedure:}

Impact evaluation level was used to assess short-term effect, immediately after the PEP, on patients' knowledge, beliefs, and practices immediate. One assessment was approached through pre-designed pilot-tested interview questionnaire. The questionnaire was designed to elicit the following information: 
1-Personal and socioeconomic data.

2-Patients' knowledge about

peridontitis consisted of 27 yes/no statements pertaining to patients' knowledge about definition of peridontitis, its signs and symptoms, risk factors, causes and complications of peridontitis, types of treatment options, uses of tooth brushing, flossing, and mouth wash.

Each question was scored from (0-1). The total score ranged from (0-27). Standardized item alpha reliability coefficient (ARC) was 0.797 for knowledge instrument.

\section{3-Patients' beliefs about peridontitis} using the principles of HBM: this was measured through Likert type statements scored from 1 to 3 with higher score for higher belief (p. susceptibility, p. seriousness, and $p$. benefits) and higher $p$. barriers. These included:

a- Perceived susceptibility (P. susceptibility): was measured through 4 items that all persons are susceptible to gingivitis, peridontitis and plaque can form in all mouths, plaque layer can lead to calculus, and simple gingivitis if not treated leads to peridontitis. The score ranged from 4 to $12, A R C=0.768$

b- Perceived seriousness (P. seriousness): included 2 items that peridontitis is a serious disease causing loss of teeth, and may lead to dental abscess. The total score ranged from 2 to $6, \mathrm{ARC}=0.774$

c- Perceived benefits of compliance with treatment and keeping appointment: consisted of eight items entailing good oral hygiene, teeth brushing, flossing, professional cleaning may be beneficial for some cases, some types of mouth wash help prevent plaque formation and treatment, keeping appointment and compliance keep healthy gum and lead to complete recovery and surgery may be the best treatment for advanced cases after oral hygiene, and professional cleaning. The total score range was $8-24, \mathrm{ARC}=0.737$.

d- Perceived barriers to compliance with treatment and keeping appointment: included six factors pertaining to the 
high cost of floss and treatment, fear of pain, professional cleaning is lost of time, long waiting time at clinic, and forgetfulness. The total score range was 6-18. $\mathrm{ARC}=0.835$. $\mathrm{A}$ total $\mathrm{HBM}$ score was calculated by summing up $\mathrm{p}$. susceptibility

p. seriousness,

p. benefits, and minus p. barriers. The total score range was20-60.

4-The patient's behavior scale: included 14 multiple choice items regarding compliance with treatment, oral health practice, and keeping appointment, regular use of drug, proper teeth brushing, brushing two times daily, flossing daily, brushing tongue daily, eating balanced diet containing vegetables and fruits, decreasing snacking between meals, and seeking dentist help for any unusual periodontal symptoms. Each item was scored from 1 to 4 with higher score for the best oral practice as judged by two professors of dentistry. The total score ranged from 14 to 56 .

Data analysis: Data were analyzed using SPSS program (version 11). The influence of PEP on knowledge, beliefs, and practice scores was assessed by examining the mean scores of data of the intervention and control groups using t-test. Chi-square test and a stepwise multiple regression model procedure were employed.

\section{RESULTS}

The intervention and the control groups were comparable with no statistically significant difference as regard most sociodemographic factors. Concerning age it was found that $80.6 \%$ of the intervention group belonged to age group (20-years) compared to $56.3 \%$ of the control. It was also noticed that $68 \%$ of the intervention group patients had (10-12 years) of education compared to $47.6 \%$ of the control group. These differences were statistically significant, $\chi^{2}=(=14.33,9.98$ $\mathrm{p}<0.01$ ), respectively, Table 1 .

Table 2 shows that the mean knowledge score of the intervention group was significantly higher than that of the control group $(t=-6.352, p<0.01)$. Regarding health belief model (HBM) components, the table demonstrates that mean scores of 
perceived seriousness, benefits, and intervention group, the 14 variables barriers scores were significantly higher entered and only 3 variables were among the intervention group than those of selected: total knowledge, patient work, the controls $(t=-5.207,-7.175,1.715)$, and total HBM score. The three variables respectively. The table also points out that mean score of perceived barriers was significantly lower among the intervention group than those of the controls $(t=1.715)$

The stepwise multiple regression in table 3 shows that factors entering the regression of models $(1,2)$ of patients' practice were: sociodemographic (age, sex, marital status, family size, family head education, education of the patients, patient's work, and residence), total knowledge score, perceived susceptibility, perceived seriousness, perceived benefits, and perceived barriers, total HBM and only 4 out of 14 studied factors have predicted patients' practice of the control group: total knowledge, perceived benefits, patient work, and family size. The four variables together explained $24 \%$ of the variation of the practice score, patients' work positively predicted patients' practice, i.e., not working had poor practice. Among the explained $30.3 \%$ of the variance of the practice score. Patients' work negatively predicted patients practice, i.e., not working had good practice.

\section{DISCUSSION}

Tubaishat et al., 2005 found thatoverall, the level oral health knowledge was low, and oral health practice was poor. ${ }^{21}$ This result coincides with that of the present study, where the mean knowledge score of the control group was only $18.38 \pm 4.448$ (total score $=27$ ) and the mean practice score of the control group was only $37.51 \pm 8.13$ (total score=56) denoting low knowledge and poor practice. Similarly, Al-Otaibi and Angmar-Masson 2004 in the city of Makkah found that knowledge and awareness of oral health is very low. Despite the availability of free dental care at public health centers, $89 \%$ of the participants at the military center and 
$54 \%$ at university center sought only (benefits). Similarly, that study revealed emergency care. ${ }^{22}$ Wang et al., $2007^{23}$ that the patients had moderately high concluded from their study that most benefits as its mean score among control Chinese have no knowledge of common group was $18.18 \pm 2.98$ (the score range: periodontal prevention and treatment $8-24) \cdot{ }^{24}$

and very few have regular dental care. ${ }^{23}$

Brown $1994^{26}$ reviewed research in On the other hand, Vignarajah²4 1997 found that the majority of young people were able to correctly identify signs of periodontal disease. However, they incorrectly defined the meaning of plaque. Students claimed good oral hygiene behavior and willingness to receive treatment from dentists. $74 \%$ of adolescents went to dentist at sometime.

Cost and fear were found to be the common barriers for regular dental attendance. ${ }^{24}$ Also, the present study showed that the patients had moderate barriers as its mean score among control group was $8.31 \pm 1.86$ (the score range: 6-18). Bader et al., $1989^{25}$ concluded that patients' knowledge was not perfect. Patients had a strong positive belief about keeping their teeth for lifetime health education and health promotion, in the period of 1982 to 1992 , a total of 57 studies evaluating the effectiveness of intervention to alter individuals' behavior related to dental health. ${ }^{26}$ Combining the results of these 57 studies with descriptive articles published over the same period, it appeared that health education can result in improvements in objective measures of dental health behavior and oral health measures, but has only limited success in changing attitudes toward dental issues and achieves only short-term gain in knowledge. ${ }^{26}$ Renz et al., $2007^{27}$ concluded that there is tentative evidence from studies that psychological approaches to behavior management can improve oral hygiene related behaviors. ${ }^{27}$

in contrast, the present study shows that patients' mean knowledge; p. seriousness, 
p. benefits, and p. barriers scores were evidence that tooth brushing prevents significantly higher in the intervention periodontal disease is equivocal. ${ }^{29}$ group. Mean behavior and p. susceptibility Hugoson et al., $2007^{32}$ concluded that their scores were higher in the intervention study confirms the efficacy of three group but didn't reach the significant level. different programs in reducing Grocholewicz 1999 concluded that the supragingival plaque and gingival results showed a distinct improvement of inflammation. Professional cleaning health awareness among examined provides no clinical benefit beyond that children and less improvement in oral derived form individual and group-based hygiene. ${ }^{28}$ This may be due to that health education. ${ }^{32}$ The study of Philippot demonstrating behavioral change takes et al., $2007^{33}$ indicated that behavioral time and success is often judged by intervention is more effective than a positive results. ${ }^{26}$ While, the present study classical intervention based on information outcome evaluation measure was done immediately after the program, the patients couldn't apply his knowledge into practice but they could maximize their practice according to their understanding from the program. Other studies concluded that there have been impressive improvements in oral knowledge, attitudes and behavior after health education programs. ${ }^{29-31}$

The majority of oral disease is related to lifestyle and reducing these mostly chronic diseases rely much on changing behavior. For example, the and training about prophylactic techniques and in bringing most patients to normal level of plaque indices.

Facts are an important part of the message but must be complemented by reflection and consideration of how the receiver understands it. ${ }^{34}$ This statement is in line with the results of the current study, where knowledge was a predictor of practice of both control and intervention groups. Hugoson et al., $2007^{32}$ found that knowledge of dental diseases (caries, gingivitis and peridontitis) was a predictor 
to good gingival health. ${ }^{32}$ On the other hand, Aedman et al., $2006^{34}$ mentioned that facts are not enough to induce adolescents to change behavior. ${ }^{34}$ Also Grocholewicz 1999 found that there is a discrepancy between children knowledge and prophylactic health behavior. ${ }^{28 .}$

The present study reveals that total perceived benefits was a predictor of behavior among control group. Similarly, some studies pointed out that individuals who had favorable beliefs about the importance of preventive behavior for oral health had had better oral hygiene ${ }^{35,36}$ and less periodontal disease. ${ }^{36}$

The present study demonstrates that work of patient was a positive predictor of patient's behavior among the control and a negative one among intervention group, Table 3. The patient's work, a possible surrogate of socioeconomic status, may also be linked to health care practice among the control group. Similarly, Grocholewicz 1999 noticed that patients' socioeconomic status had influence on building good hygiene. ${ }^{28}$ While, after the intervention, the patients understand the good practice and its benefits and accordingly report the right practice regardless of their work.

\section{CONCLUSION AND RECOMMENDATIONS}

It could be concluded that in spite of one session the hospital based-peridontitis education program, it proved to have positive effects on peridontitis patients' knowledge, and beliefs ( $p$. seriousness, $p$. benefits, and $\mathrm{p}$. barriers).

The study recommended the replication of such PEP and a more long term one among many peridontitis patients to have more improvement in patients' knowledge, beliefs and bringing improvement in $p$. susceptibility and practice, especially compliance and oral hygiene. Finally, in spite of that the present study showed number of important factors affecting patients' practice, yet much of their variance was not explained. Therefore, continued exploration of other predictors is an essential action. 
Table 1: Distribution of the sample according to their sociodemographic characteristics

\begin{tabular}{|c|c|c|c|c|c|c|}
\hline \multirow{3}{*}{$\begin{array}{l}\text { Sociodemographic } \\
\text { characteristics }\end{array}$} & \multicolumn{2}{|c|}{ Control } & \multicolumn{2}{|c|}{ Intervention } & \multicolumn{2}{|c|}{ Total } \\
\hline & \multicolumn{2}{|c|}{$N=103$} & \multicolumn{2}{|c|}{$N=103$} & \multicolumn{2}{|c|}{$\mathrm{N}=\mathbf{2 0 6}$} \\
\hline & No & $\%$ & No & $\%$ & No & $\%$ \\
\hline \multicolumn{7}{|l|}{ Sex } \\
\hline Males & 91 & 88.3 & 87 & 84.5 & 178 & 86.4 \\
\hline Females & 12 & 11.7 & 16 & 15.5 & 28 & 13.6 \\
\hline \multicolumn{7}{|l|}{ Age* $^{*}$} \\
\hline$<20$ year & 33 & 32.0 & 16 & 15.5 & 49 & 23.7 \\
\hline $20-$ & 58 & 56.3 & 83 & 80.6 & 141 & 68.5 \\
\hline $40-+$ & 12 & 11.7 & 4 & 3.9 & 16 & 7.8 \\
\hline \multicolumn{7}{|l|}{ Marital status } \\
\hline Single & 69 & 67.0 & 75 & 72.8 & 144 & 69.9 \\
\hline Ever married & 34 & 33.0 & 28 & 27.2 & 62 & 30.1 \\
\hline \multicolumn{7}{|l|}{ Family size } \\
\hline$<5$ person & 18 & 17.5 & 14 & 13.6 & 32 & 15.6 \\
\hline $5-$ & 62 & 60.2 & 67 & 65.0 & 129 & 62.6 \\
\hline $10_{+}$ & 23 & 22.3 & 22 & 21.4 & 32 & 15.6 \\
\hline \multicolumn{7}{|c|}{ Education of family head } \\
\hline$\leq 9$ years & 22 & 21.4 & 33 & 32.0 & 55 & 26.7 \\
\hline $10-$ & 32 & 31.1 & 23 & 22.4 & 55 & 26.7 \\
\hline$>12$ & 49 & 47.6 & 47 & 45.6 & 96 & 46.6 \\
\hline \multicolumn{7}{|l|}{ Education of patient ${ }^{*}$} \\
\hline$\leq 9$ years & 26 & 25.2 & 20 & 19.4 & 46 & 22.3 \\
\hline $10-$ & 49 & 47.6 & 70 & 68.0 & 119 & 57.8 \\
\hline$>12$ & 28 & 27.2 & 13 & 12.6 & 41 & 19.9 \\
\hline \multicolumn{7}{|l|}{ Work of patient } \\
\hline Not Working & 30 & 29.1 & 21 & 20.4 & 51 & 24.7 \\
\hline Student & 50 & 49.5 & 51 & 50.5 & 101 & 49.1 \\
\hline Employee & 23 & 22.3 & 31 & 30.1 & 54 & 26.2 \\
\hline \multicolumn{7}{|l|}{ Type of house } \\
\hline Apartment & 25 & 24.3 & 25 & 24.3 & 50 & 24.3 \\
\hline Villa & 78 & 75.7 & 78 & 75.7 & 156 & 75.7 \\
\hline
\end{tabular}


Table 2: Impact of glaucomatous education program on peridontitis' Knowledge, beliefs (HBM: P. susceptibility, seriousness, benefits and barriers, and total HBM score) and Practices of Peridontitis patients

\begin{tabular}{|l|c|c|c|c|}
\hline Variables & No. & Mean \pm SD & t & p \\
\hline $\begin{array}{l}\text { Total knowledge } \\
\text { Control group } \\
\text { Intervention group }\end{array}$ & 103 & $\begin{array}{c}18.38 \pm 4.448 \\
21.84 \pm 3.30\end{array}$ & $-6.352^{\star}$ & 0.001 \\
\hline $\begin{array}{l}\text { Perceived susceptibility } \\
\text { Control group } \\
\text { Intervention g }\end{array}$ & 103 & & & \\
\hline $\begin{array}{l}\text { Perceived seriousness } \\
\text { Control group } \\
\text { Intervention g }\end{array}$ & 103 & $10.01 \pm 1.66$ & $-5.478^{*}$ & 0.142 \\
\hline $\begin{array}{l}\text { Perceived benefits } \\
\text { Control group }\end{array}$ & 103 & $4.22 \pm 1.24$ & & \\
Intervention g & 103 & $5.04 \pm 0.10$ & $-5.207^{\star}$ & 0.001 \\
\hline $\begin{array}{l}\text { Perceived barriers } \\
\text { Control group }\end{array}$ & 103 & $18.18 \pm 2.98$ & $-7.175^{\star}$ & 0.038 \\
Intervention g & 103 & $20.94 \pm 2.54$ & & \\
\hline $\begin{array}{l}\text { Total HBM score } \\
\text { Control group } \\
\text { Intervention g }\end{array}$ & 103 & $8.88 \pm 2.83$ & & \\
& 103 & $8.31 \pm 1.86$ & $1.715^{*}$ & 0.002 \\
\hline $\begin{array}{l}\text { Total practice score } \\
\text { Control group } \\
\text { Intervention g }\end{array}$ & 103 & $22.52 \pm 4.96$ & -8.45 & 0.061 \\
& 103 & $27.86 \pm 4.06$ & & \\
\hline
\end{tabular}

${ }^{*}=p<0.01$

Table 3: Summary of Stepwise Multiple Regression Analysis for peridontitis Practices for control $(\mathrm{N}=103)$ and intervention group $(\mathrm{N}=103)$

\begin{tabular}{|l|c|c|c|c|c|c|c|}
\hline Predictors & B & Beta & SE & T & $\mathbf{R}^{2}$ & F ratio & P \\
\hline $\begin{array}{l}\text { Models 1, 2 total } \\
\text { practice score: }\end{array}$ & & & & & & & \\
\hline $\begin{array}{l}\text { Control group } \\
\text { Constant }\end{array}$ & 10.434 & & 5.243 & 1.99 & 0.24 & 7.735 & 0.049 \\
\hline Total knowledge & 0.525 & 0.287 & 0.166 & 3.159 & & & 0.002 \\
\hline Total benefits & 0.597 & 0.218 & 0.251 & 2.382 & & & 0.019 \\
\hline Work & 1.159 & 0.194 & 0.526 & 2.203 & & & 0.03 \\
\hline Family size & -0.216 & 0.194 & 1.145 & 2.16 & & & 0.033 \\
\hline Intervention group & & & & & & & \\
\hline Constant & 12.097 & & 4.668 & 2.592 & 0.303 & 14.363 & 0.011 \\
\hline Total knowledge & 0.513 & 0.239 & 0.210 & 2.447 & & & 0.016 \\
\hline Patient work & -1.295 & -0.212 & 0. & -2.416 & & & 0.018 \\
\hline Total HBM & 0.716 & 0.411 & 0.176 & 4.076 & & & 0.000 \\
\hline
\end{tabular}

Factors entering the regression:

Models 1\&2: Sociodemographic variables (age, sex, marital status, family size family head education, education of the patient, patient work, residence), total knowledge score, p. susceptibility, p. seriousness, p. benefits, p. barriers, and total HBM. 


\section{REFERENCES}

1. Al-Tamini S, Peterson PE. Oral health situation of schoolchildren, mothers, and schoolteachers in Saudi Arabia. Int Dent J. 1998; 48 (4): 180-6.

2. Al-Mutawa S, Al-Duwairi W, Honkala E, Honkala S, Shyama M. The trend of dental caries experience of children in Kuwait. Dental News. 2002; 9: 9-13.

3. Bradnock G, Whit DA, Nuttall NM, Morris AJ, Treasure ET, Pine CM. Dental attitudes and behaviors in1998 and implication for the future. Br Dent J. 2001; 190: 228-32.

4. Rimondini L, Zolfanilli B, Bernardi F, Bez C. Self preventive oral behavior in an Italian university student population. J Clin Perdontol. 2001; 28: 207-11.

5. Stenberg P, Hakansson J, Akerman S. Attitude to dental health and care among 20 to 25-year-old Swedes: results from a questionnaire. Acta Odontol Scand. 2000; 58: 102-6.

6. Astrom AN, Samdal O. Time trends in oral health behavior among Norwegian adolescents. Acta Odontol Scand. 2001; 59: 193-200.

7. Kulak-Ozkan Y, Ozkan Y, Kazazoglu E, Arikan A. Dental caries prevalence, tooth brushing and periodontal status in 150 young people in Istanbul: A pilot study. Int Dent J. 2001; 51: 451-6.

8. Kassak KM, Dagher R, Doughan B. Oral hygiene and lifestyle correlates among new undergraduate university students in Lebanon. J Am Coll Health. 2001; 50: 15-20.
9. Vigild M, Peterson PE, Hadi R. Oral health behavior of 12-year-old children in Kuwait. Int $\mathrm{J}$ Paediatr Dent. 1999; 9: 23-9.

10. Behbehani JM, Shah NM. Oral health in Kuwait before the Gulf war. Med Principles Pract. 2002; 11: 3643.

11. Holst D, Schuler A, Grytten J. Future treatment needs in children, adults and the elderly. Community Dent Oral Epidemiol. 1997; 25:113-8.

12. Sogaard AJ, Grytten J, Holst D. Recent changes in health related dental behaviors in Norway. Community Dent Oral Epidemiol. 1991; 19:241-5.

13. Peterson PE. Dental health behavior among 25-44-year-old Danes. Scand J Prim Health Care. 1986; 4:51-7.

14. Heloe LA, Holst D, Rise J. Development of dental status and treatment behavior of Norwegian adults 1973-85. Community Dent Oral Epidemiol. 1988; 16:52-7.

15. Ashley FP. Role of dental health education in preventive dentistry. In: Murray JJ. Prevention of dental disease. Oxford: Oxford university Press; 1996. 406-414

16. Freeman $R$, Maizels J, Wyllie $M$, Sheiham A. The relationship between health-related knowledge, attitudes and dental health behaviors in 14-16 -year-old adolescents. Community Dent Health. 1993; 10:397-404.

17. Kay EJ, Locker D. A systematic review of the effectiveness of health promotion aimed at improving oral health. Community Dent Oral Epidemiol. 1998; 26:132-44. 
18. Woodgroove J, Cumberbatch G, Gylbier S. Understanding dental attendance behavior. Community Dent Health. 1987; 4:215-21.

19. Hamilton ME, Coulby WM. Oral health knowledge and habits of senior elementary health school students. J Publ health Dent. 1991; 51:212-8.

20. Anonymous. Health Education Behavior Models and Theories - A Review of the Literature-Part 1. Available from: http//msucares.com/health/appa1.ht $\mathrm{m}$.

21. Tubaishat RS, Darby MI, Baum DB, Box CE. Use of miswak versus toothbrushes: oral health beliefs and behaviors among sample of Jordanian adults. Int $\mathrm{J}$ Dent Hyg. 2005; 3(3):126-6.

22. Al-Otaibi M, Angmar-Masson B. Oral hygiene and oral health awareness among urban Saudi Arabian. Oral Health Prev Dent. 2004; 2(4):389-96.

23. Wang QT, Wu ZF, Wu YF, Shu R, Pan YP, Xia JL. Epidemiology and preventive direction of periodontology in China. $\mathrm{J}$ Clin Periodontol. 2007; 34(1): 946-51.

24. Vignarajah S. Oral health knowledge and behaviors and barriers to dental attendance of school children and adolescents in the Caribbean island of Antigua. Int Dent J. 1997; 47930: 167-72.

25. Bader JD, rosier G, Mc Fall WT, Ransey DL. Dental patients' knowledge and beliefs about periodontal disease. Community Dentistry \& Oral Epidemiology. 1989; 17(2):60-4.
26. Brown LF. Research in dental health education and health promotion: $A$ review of the literature. Health Education and Behavior. 1994; 21(1):83-102.

27. Renz A, Ide $M$, Newton $T$, Robinson PG, Smith D. Psychological interventions to improve adherence to oral hygiene instructions in adults with periodontal diseases. Cochrane Database Syst Rev. 2007 Apr 18 ;(2):CD005097.

28. Grocholewicz K. the effect of prophylactic-educational programs on oral hygiene, periodontum and caries in school children during a 4year observation. Ann Acad Med Stetin. 1999; 45;265-83.

29. Petersen PE, Kwan S. Evaluation of community based health promotion and oral disease prevention - WHO recommendation for improved evidence in public health. Community Dental Health. 2004; 21(supplement): 319-29.

30. Puska P. Do we learn our lessons from the population-based intervention? Journal of Epidemiology and Community Health. 2000; 54; 562-3.

31. Petersen PE. Evaluation of dental prevention program for Danish chocolate workers. Community dentistry and Oral Epidemiology. 1989; 17: 53-9.

32. Hugoson A, Lundgren D, Asklow B, Brogklint G. Effect of three different health preventive programs on young adult individuals: a randomized, blinded, parallel group, controlled evaluation of oral hygiene behavior on plaque and gingivitis. J Clin Periodontol. 2007; 34(5):407-15. 
33. Philippot P, Lenoir N, D'Hoore W, Bercy $P$. Journal of Clinical Periodontology. 2005; 32(60):653-8.

34. Aedman E, Ringberg C, Gabre P. Knowledge of and attitude to oral health and oral disease among young adolescents in Sweden. Swed Dent J. 2006; 30940:147-54.

35. Astrom AN, Jackson W, Mwangosi IE. Knowledge, beliefs and behavior related to oral health among Tanzanian and Ugandan teacher trainees. Available from: anne.nordrehaug@cih.uih.no 1999

36. Broadbent JM, Thomson WM, Poulton R. Oral health beliefs in adolescence and oral health in young adulthood. J Dent Research. 2006; 85; 339-43. 